\title{
HERRAMIENTAS PARA EL ANÁLISIS \\ MORFOLÓGICO DE FABRA I COATS EN BARCELONA. PROCESAMIENTO DE IMÁGENES A PARTIR DE LA TECNOLOGÍA DE ESCÁNER LÁSER TERRESTRE*
}

Juan Manuel Corso Sarmiento**

Universidad Politécnica de Catalunya, España

Josep Roca Cladera***

Universidad Politécnica de Catalunya, España

Recibido: 30 julio 2013

Aprobado: 20 septiembre 2013

Levantamiento TLS, perspectiva nube de puntos Fabra i Coats

Fuente: Elaboración propia de los autores.

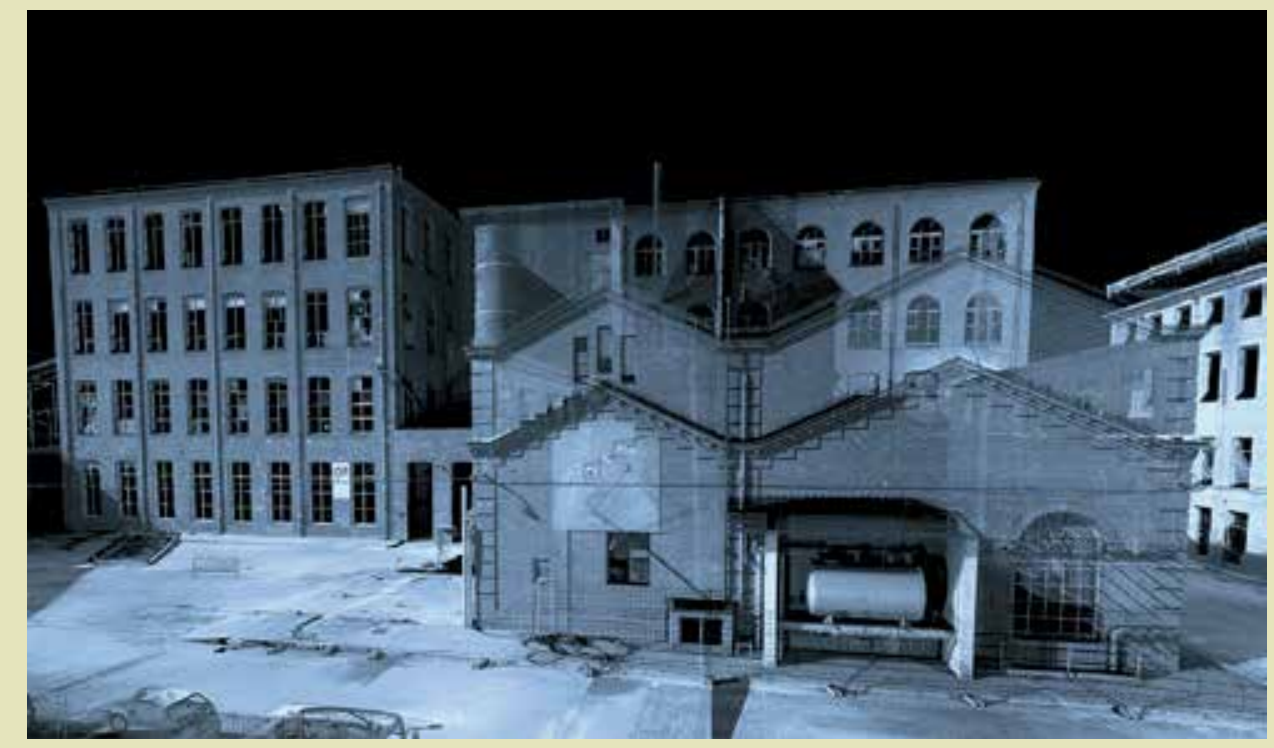

\section{RESUMEN}

Este artículo parte de la tesis en desarrollo: CORSO, J. Procesamiento de Imágenes LiDAR Terrestre. Análisis, selección y síntesis de la información del Escáner Láser Terrestre como bases de datos 2Dy 2.5D. Doctorado en Gestión y Valoración Urbana y Arquitectónica, Universidad Politécnica de Catalunya.

** Arquitecto, Candidato a Ph.D. Responsable técnico del Laboratorio de Modelización Virtual de La Ciudad (LMVC) en la Universidad Politécnica de Catalunya, España.

E-mail: juan.manuel.corso@upc.edu

*** Doctor Arquitecto. Director del "Centre de Política de Sòl i Valoracions" (CPSV). Director del Departamento de Construcciones Arquitectónicas I en la Universidad Politécnica de Catalunya, España.

E-mail: josep.roca@upc.edu

En este artículo se plantea una metodología para el análisis morfológico de fachadas patrimoniales a partir de un levantamiento con la Tecnología de Escáner Láser Terrestre TLS, tanto desde un punto de vista tridimensional, como del desdoblamiento de las fachadas en $2 \mathrm{D}$ y $2.5 \mathrm{D}$, manteniendo en todo momento el control de la información de partida, su transformación para ser explotada en diversos formatos, con objetivos específicos, a diferentes escalas, desde el nivel arquitectónico con deformaciones de fachadas y análisis de texturas hasta la identificación del relieve de los materiales, como una herramienta que amplía la información para ser interpretada por especialistas de diversas disciplinas relacionadas a la conservación del bien patrimonial. El caso del estudio propuesto, es un ejemplo destacado del patrimonio Industrial de Cataluña, las calderas de Fafra i Coats, las cuales fueron documentadas utilizando un escáner láser Faro Focus3D.

\section{PALABRAS CLAVE}

Escáner Láser Terrestre, Patrimonio, Tecnologías de Documentación, Procesamiento de Imágenes. 


\section{TOOLS FOR THE MORPHOLOGICAL ANALYSIS OF FABRA I COATS IN BARCELONA. IMAGES PROCESSING FROM TERRESTRIAL LASER SCANNER TECHNOLOGY}

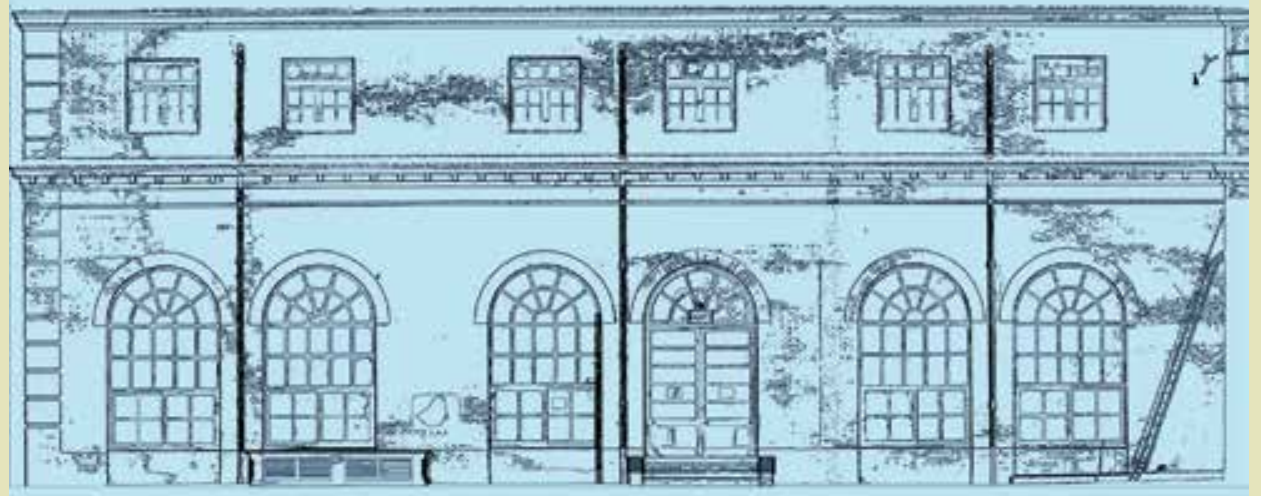

Análisis de textura Second Moment de modelo digital de superficie sin deformación, basada en una matriz Co-ocurrence Fuente: Elaboración propia de los autores.

\begin{abstract}
This article presents a methodology for the morphological analysis of heritage facades, from a survey with the TLS Terrestrial Laser Scanner Technology, both from a threedimensional point of view and the splitting of the facades in $2 \mathrm{D}$ and $2.5 \mathrm{D}$, keeping at all times the control of the starting information, its transformation to be exploited in various formats, with specific goals, at different scales, from the architectural level with deformed facades and analysis of textures, to the identification of the relief of materials, as a tool that extends the information to be interpreted by specialists in various disciplines related to the conservation of the heritage. The proposed case study is an outstanding example of industrial heritage in Catalonia, the boilers of Fabra i Coats, which were documented using a Faro Focus3D laser scanner.
\end{abstract}

\section{KEYWORDS}

Terrestrial Laser Scanning, Heritage, Documentation Technology, Image Processing. 


\section{INTRODUCCIÓN}

El registro es una responsabilidad primordial de todos los implicados en los procesos de conservación, no sólo como una obligación ética para la posteridad, sino también como fuente de beneficios inmediatos en términos de planificación de proyectos, comunicación interdisciplinar y evaluación de los resultados (Letellier, 2007). Todos los implicados en la conservación y entendimiento del lugar patrimonial, deben tener acceso a la información existente y a generar documentaciones que deben ser preservadas y disponibles para otras personas.

Este proceso se ve limitado por la comunicación entre las diferentes disciplinas involucradas en el proceso de conservación del patrimonio, puesto que no hay estándares uniformes de registro, de documentación y de gestión de la información. Por ello, se plantea en este artículo una metodología de segmentación y georreferenciación (Lerma, 2008) de la información TLS, que permita sobreponer bases de datos y análisis posteriores del bien patrimonial, de tal forma, que se pueda identificar patologías o modificaciones del edificio si se realizan estudios en diferentes fechas, sin ser una limitante la escala en la que se trabaja la información.

Lo anterior, permitiría que un registro arquitectónico pueda ser profundizado con la documentación que posibilitaría la restauración del edificio, o el estudio estratigráfico de la fachada desde el punto de vista arqueológico, teniendo en cuenta que se considera el registro arquitectónico como instrumental, mientras que la documentación del material y las tipologías de degradado son procesos manuales-visuales "naked eye".

En la actualidad, los procesos manuales-visuales están siendo modificados por las nuevas tecnologías, nos permiten sobrepasar la variante geométrica e incluso llegar a manipular información científica sobre los materiales como pérdida o alteración material de un objeto en el tiempo (Simon, 2010). Ejemplo de ello, son los trabajos desarrollados por Sithole (2008) y Willis (2010) en términos de detección de materiales de fachada; Abellan (2010) en términos de cambio de material y desgaste del mismo; Lindstaedt (20I I) y Al-Neshawy (2009) en relación a patologías por delaminación o deformaciones de fachadas.

\section{BARCELONA INDUSTRIAL, CASO DE ESTUDIO DE FABRA I COATS}

En el siglo XIX Catalunya fue un protagonista destacado en la Revolución Industrial, dejando su huella en la historia, cambiando de ser una estructura de producción artesanal a una organización de trabajo vinculado a la fábrica. Con un progreso productivo y urbanístico intenso y rápido, al punto que muchos edificios industriales dejaron de existir; otros prosiguen su abandono hasta nuevo uso o destrucción y otros han tenido la suerte de haber sido rehabilitados. Estos espacios forman parte de la identidad de Catalunya, teniendo en cuenta tanto la memoria colectiva, la urbana y la arquitectónica.

Uno de los ejemplos más destacados de estos procesos de rehabilitación es el complejo industrial de Fabra I Coats, catalogado Patrimonio Arquitectónico de Barcelona con el nivel C (Aparicio 20I I), siendo un edificio que reúne valores históricos, artísticos y estéticos, considerados relevantes en el sector urbano donde se emplaza; es una hilatura del siglo XIX que forma parte del antiguo municipio de Sant Andreu del Palomar, construido entre los años 1890 y 1955 . El ayuntamiento de Barcelona lo adquirió en el 2005, convirtiendo sus naves principales en un centro cultural de referencia; se concibe como el proyecto emblemático 
del programa "Fàbriques de Creació". Un espacio concebido para enriquecer el barrio en el cual se localiza, enfocado a la creación, la práctica y la difusión artística multidisciplinar.

El levantamiento de este bien patrimonial se realizó con la Tecnología Escáner Láser Terrestre (TLS) Faro Focus3D. Se centró en una de las torres de la nave central del complejo industrial, construida entre 1919-1929. Constituida por las naves de calderas, la central eléctrica y de bombeo, estas últimas dos centrales se ubican en la planta baja de un edificio de cuatro plantas. El conjunto industrial posee una estructura de ladrillo visto, con pilares y jácenas de perfilaría laminada inglesa, como se ve en la Figura I, render de nube de puntos.

\section{LEVANTAMIENTO CON ESCÁNER LÁSER TERRESTRE}

Como paso previo al registro de la nube de puntos, se plantearon las limitantes técnicas en relación a la complejidad del levantamiento por realizar, teniendo en cuenta los elementos de menor tamaño que se pueden encontrar en edificios industriales, estableciendo con ello los ajustes y alcance del registro final, teniendo en cuenta la densidad de medidas necesarias para todos los elementos, la distancia óptima para la captura, teniendo en cuenta los ángulos de incidencia y de oclusión, considerando las características del equipo utilizado.

El escáner utilizado se basa en la diferencia de fase, el Faro Focus3D permite un rango de captura amplio $360^{\circ} \mathrm{H}^{*} 305^{\circ} \mathrm{V}$, con una precisión de $0.6 \mathrm{~mm}$ a $10 \mathrm{~m}, 0.95 \mathrm{~mm}$ a 25 $\mathrm{m}$ (con una longitud de Honda de $905 \mathrm{~nm}$ ), con un error sistemático de $\leq \pm 2 \mathrm{~mm}$ a 25 $\mathrm{m}$, aparte de la información de intensidad reflejada del láser, se capturan fotografías con información de color RGB que se asignan a los puntos, mediante una cámara integrada de 70 megapíxeles. La resolución por posición de escaneo de Fabra I Coats fue de I/8 con 4x de calidad, esta calidad hace referencia al número de comprobación que es para reducir el error del levantamiento, obteniendo por posición en promedio 38 millones de medias, en total se realizaron 82 posiciones, obteniendo un modelo final de 1.300 millones de puntos.

Destacamos en el proceso de optimización de base de datos la unión entre posiciones, al ser un reto, por la complejidad del edificio, la estrechez de algunos espacios, la poca visibilidad entre posiciones y la resolución con la que se requería el modelo final, en el cual era necesario ver hasta las cuerdas del techo, sin que estas se vieran duplicadas por errores de unión, en un número considerable de posiciones, en espacios interconectados. Para ello, se utilizó el método de unión ICP descrito a continuación.

\section{Método de unión de nubes de puntos ICP Iterative Closest Point}

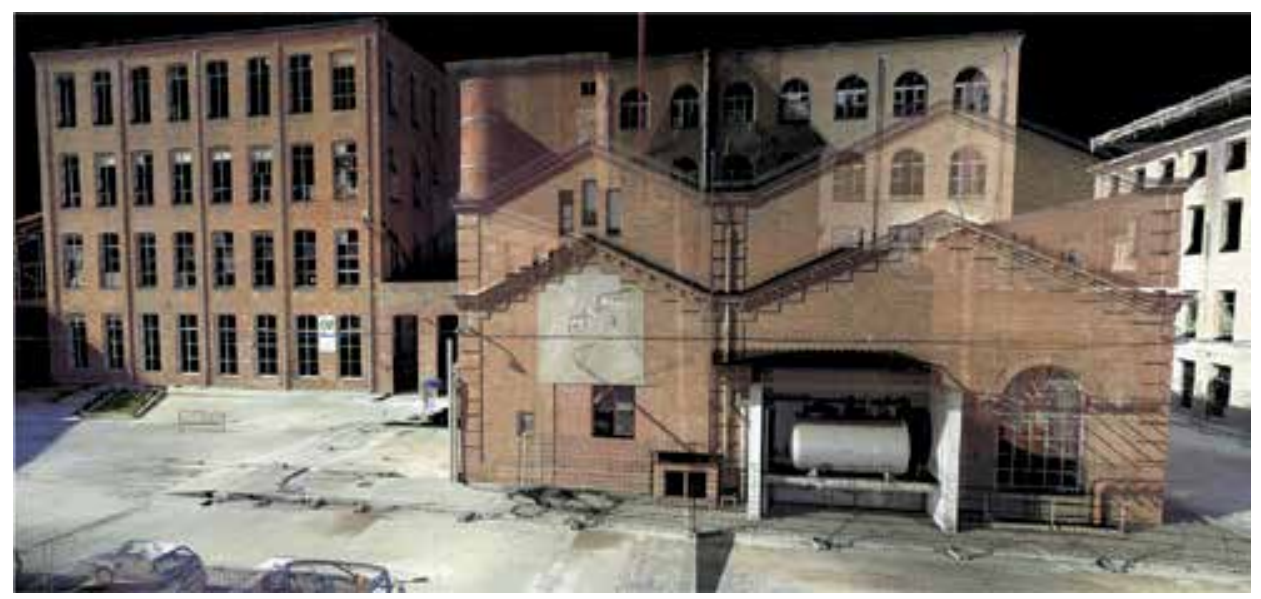

Figura I. Levantamiento TLS, perspectiva nube de puntos Fabra i Coats Fuente: Elaboración propia de los autores. 
El registro ICP es posible independientemente de la naturaleza de los datos geométricos (puntos, curvas de diferentes grados de las superficies). Se basa en minimizar la distancia entre dos nubes, punto a punto, por medio de iteraciones sucesivas. Las principales desventajas de este proceso en su forma original son, en primer lugar, que convergen en un mínimo que puede ser falso, especialmente cuando hay una gran cantidad de ruido en los datos y se necesita un gran número de iteraciones para la convergencia del modelo.

Aunque diversos estudios buscan encontrar valores iníciales para unir posiciones de forma automática para acelerar el proceso de unión, se sigue utilizando una variante manual al asignar puntos homólogos entre las nubes para su registro inicial, con estos puntos de partida es posible calcular una transformación rígida inicial y luego comenzar las iteraciones. Sin embargo, no siempre es posible encontrar puntos homólogos en las nubes de puntos, por lo cual se habla de "puntos de conexión", si el algoritmo no los encuentra, tratará de sustituirlos por los puntos más cercanos, ejemplo de ello es la Figura I.

\section{CLASIFICACIÓN DEL PATRIMONIO INDUSTRIAL}

Figura 2. Registro mediante el promedio de la distancia entre puntos de cada posición escaneada ICP

Fuente: Elaboración propia de los autores.

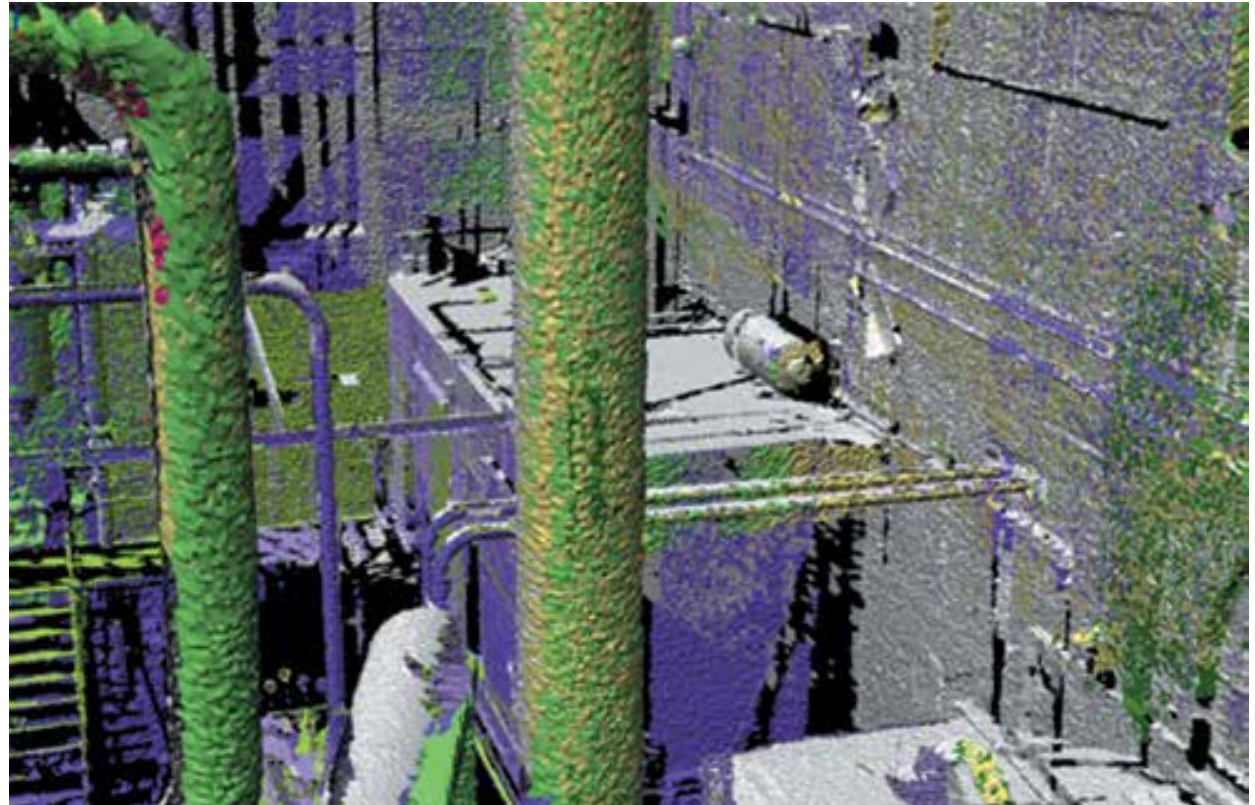

La clasificación del edificio patrimonial requiere tanto una clasificación por la forma arquitectónica, como también la evolución histórica de las maquinarias y como éstas modificaron la fábrica textil. La clasificación fue un proceso manual utilizando las herramientas de Pointools Edit.

El primer paso de la clasificación fue la limpieza de la nube, ya que las 82 posiciones tenían más de 1.400 millones de puntos, limitando exportar zonas seleccionadas del modelo. Se consideró que las intensidades menores de $0.25 \mathrm{db}$ con una tolerancia de $20 \%$, son ruido causado por una reflectancia muy alta, como son las zonas de cristales y errores causados por proximidad de objetos que generaron ruido en las proyecciones lejanas. Estos valores se determinaron mediante pruebas consecutivas sobre la selección de información del modelo y la verificación posterior, como se ve en la Figura 3. 
Figura 3. Selección por intensidad en procesos

de limpieza de la nube de puntos

Fuente: Elaboración propia de los autores.

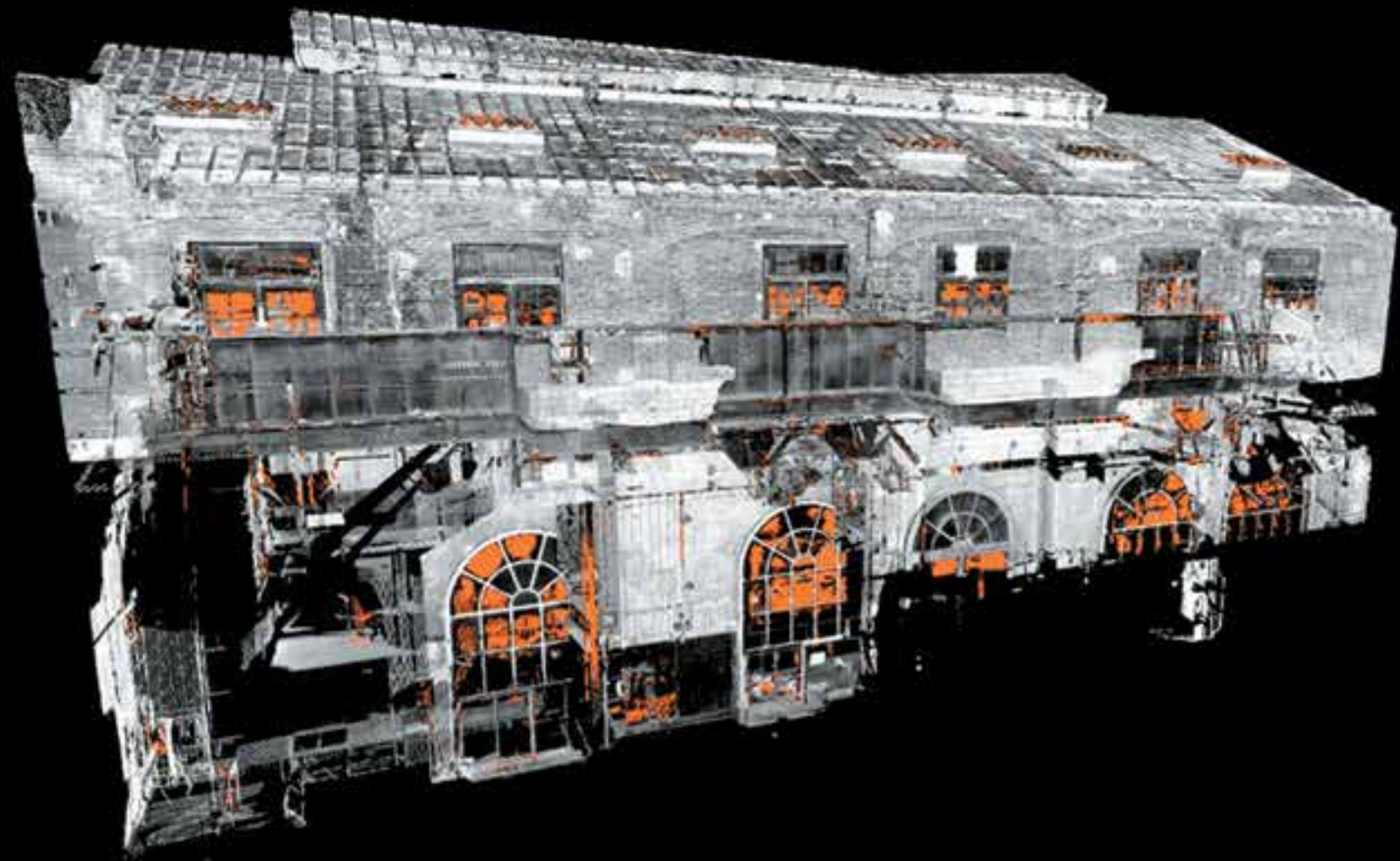

M 89 REVISTA M VOL. 10 No.2. JULIO-DICIEMBRE 2013 - FACULTAD DE ARQUITECTURA • UNIVERSIDAD SANTO TOMÁs COLOMBIA 
Por último, se realizaron procesos de limpieza automáticos con escáneres láser aéreos LIDAR (Light Detection and Ranging): filtro de puntos aislados del promedio de la nube de puntos, filtro de grupos de puntos fuera del promedio de la nube de puntos predefinido, llegando a un total de 917.111 .719 puntos como información preliminar, antes de comenzar los procesos de clasificación.

Esta clasificación comenzó con las dos calderas de carbón más antiguas (caldera N2 y N3, véase Figura 5), alimentadas a mano, posteriormente apoyadas por un sistema basado en tolvas, afirmadas por el muro portante y una estructura complementaria metálica. Estas calderas cambiaron de fuente de energía a gas, modificándolas con un quemador y un acumulador de vapor en la parte superior.

La introducción de dos nuevas calderas (calderas $\mathrm{N} 4$ y $\mathrm{NI}$ ), obligó a la ampliación de la fábrica, elevando tres metros el techo y los muros, llegando a una altura en el punto más bajo de diez metro de altura. Las nuevas calderas a gas se encuentran elevadas, respecto a las anteriores, para facilitar su limpieza. La última caldera se introdujo realizando una abertura en un muro perimetral, ya que esta máquina es de menor tamaño y no requirió el desmontaje del techo para su instalación, esta caldera es de gas y tiene un control eléctrico (caldera N5).

Los sistemas de ventilación asociados a las calderas fueron modificados con el tiempo, dejando en la parte exterior del complejo una chimenea de ladrillo y otras metálicas. Complementario a este sistema, hay dos sistemas paralelos: uno eléctrico y uno hidráulico, que servían para distribuir el vapor de las calderas como agua caliente al resto de edificios y como sistema de prevención de incendios. Estos combinados con las calderas generan una compleja trama de conductos y máquinas auxiliares, que se distribuyen por los muros y se conectan en la parte superior con las calderas.

Figura 4. Clasificación manual. Izquierda: rojo circulación, naranja cercha, amarillo tolvas almacenaje carbón, gris suelo. Derecha: Calderas N2 y N3

Fuente: Elaboración propia de los autores.

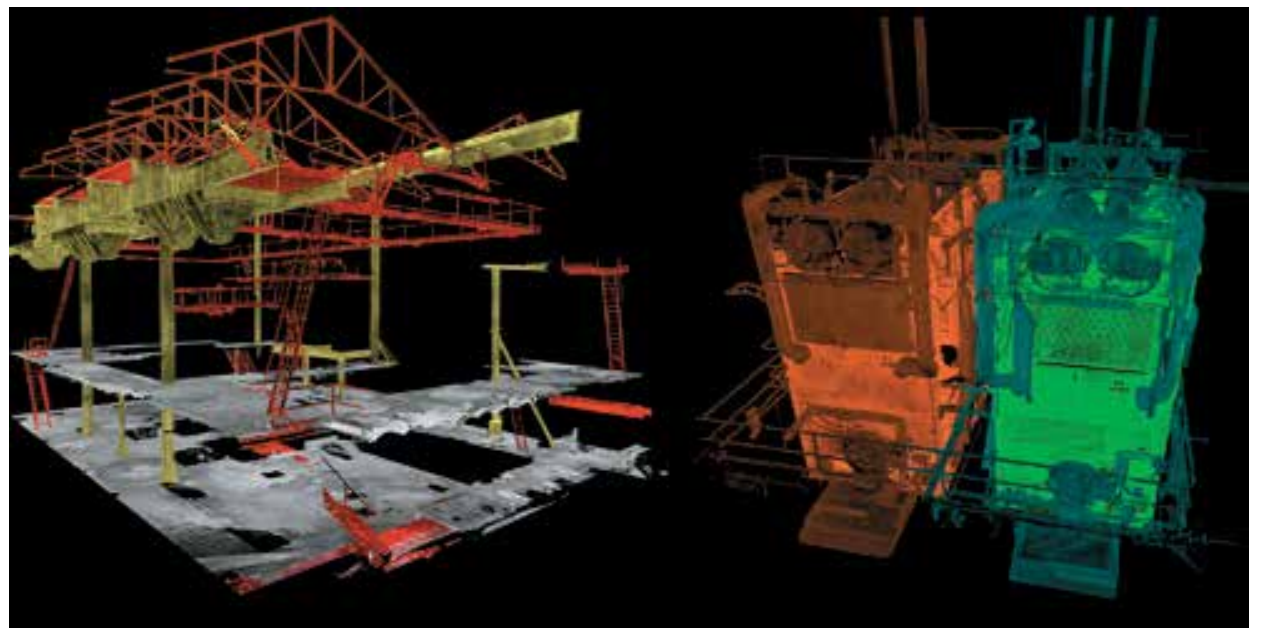

La parte arquitectónica está en estrecha relación con estas máquinas, en el nivel inferior con bodegas, los pisos superiores con una estructura metálica para la circulación (ver Figura 5) que llega hasta el punto más alto de las máquinas, el techo con sus modificaciones con una estructura de cercha metálica y una estructura del mismo material que se sigue modificando en la actualidad como refuerzo del complejo. 


\section{INFORMACIÓN GEOMÉTRICA, LA FORMA DE LA FACHADA}

Tanto los edificios patrimoniales como su contenido son objetos físicos susceptibles al deterioro, tanto de su variante constructiva y estructural, como por estar expuestos en ambientes no controlados (Simon, 2010), los mecanismos de este deterioro son amplios y pueden resultar en la alteración y pérdida de material con el tiempo.

En procesos de restauración de estos bienes, es indispensable delimitar zonas de las fachadas para cuantificar su afectación y planificar los tiempos-costos de su conservación, igualmente, en otros campos como en la arqueología se zonifican las fachadas históricas, este proceso se llama estratigrafía de fachadas, destacando la doble condición diacrónica (histórica) y sincrónica (arquitectónica) de los materiales y el edifico histórico (González-Moro, 20I I).

La cuantificación de este deterioro se puede llevar a cabo a partir de la información TLS, entendiendo que esta información es una herramienta que permite a los profesionales tomar decisiones, tanto de elementos específicos, como del conjunto del edificio; ejemplo de ello fue la intervención sobre la cercha de la cubierta de Fabra i Coats, la cual fue estudiada desde los planos generados a partir de la nube de puntos.

En la actualidad, estos procesos se llevan a cabo en su mayoría de forma visual, en algunos casos mediante orto imágenes. Consideramos que la información geométrica de la superficie de los materiales permitiría profundizar y añadir rigor a estos procesos, en los cuales la relación mortero-ladrillo es esencial, ya que estos materiales secos y húmedos permiten el estudio del deterioro o valor añadido que tienen fachadas de importancia patrimonial.

Estas intervenciones pueden realizarse a diferentes escalas y con diferentes enfoques; a continuación profundizaremos en la parte morfológica de la fachada y del material que la conforma.

\section{Deformación de la fachada}

Las fachadas analizadas se pueden considerar planas, aun si estas superficies planas presentan deformaciones en el caso de la fachada principal, producidas tanto por la presión que aplican las tolvas de carbón, como por la acción del suelo sobre el edificio.

Para cuantificar esta deformación, se analizó la fachada en relación a un plano en 3D y la verticalidad del modelo, permitiendo visualizar de forma directa su afectación pero impidiendo trabajar sobre dicho resultado, ya que el modelo 3D es muy complejo y no permite superponer sobre estas capas de información. Por ello, se desdoblan las caras del modelo 3D y se comienza a trabajar su información como imágenes de profundidad 2.5D georreferenciadas, en las cuales el valor de altura se almacena en el valor de cada píxel.

\section{Detalle geométrico de los materiales de fachada}

A continuación se plantea una metodología para resaltar las juntas de mortero de la fachada en relación al ladrillo, diferenciando resultados tanto en tres dimensiones, como en la superficie de fachada desdoblada.

Partimos del análisis de la nube de puntos en tres dimensiones, destacando la capacidad de un rápido análisis de la superficie (como se ve en la Figura 5) que corresponde a un segmento de la fachada izquierda de las calderas de Fabra i Coats, sobre la cual hay una fisura con grapas. Esta fachada ha sido alterada varias veces, ha crecido en altura al expandirse las 
Figura 5: Segmento de fachada lateral en 3D. Izquierda: modelo 3D texturizado, centro iluminación sobre el modelo. Derecha: análisis por curvatura RIMLSRobust Implicut MLS Fuente: Elaboración propia de los autores. calderas de carbón a gasoil y se hizo un hueco para pasar una caldera de eléctrica a través de esta fachada, después de una intervención se destapo la grieta y se grapó.

Sobre esta nube se aplicaron dos procesos: uno de iluminación y un análisis de curvatura, RIMLS Robust Implicut MLS, que permite ver qué puntos la superficie tiene un cambio por curvatura en un rango de $5 \mathrm{~cm}$.

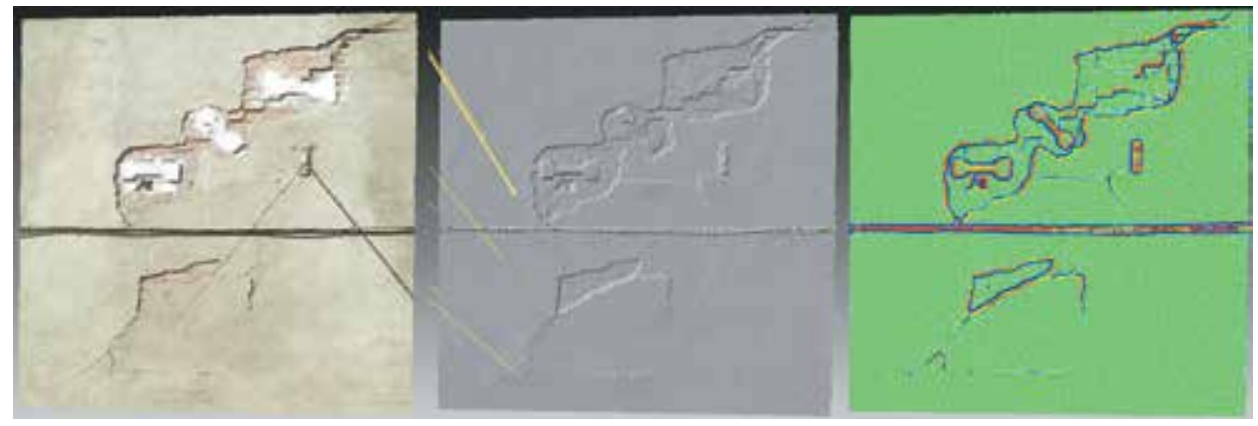

Estos análisis se pueden almacenar como un valor en la nube de puntos, pero al ser en tres dimensiones difícilmente se pueden comparar con otros datos, impidiendo superponer los análisis aplicados como capas de información. Por ello, se procedió a trasladar este proceso a imágenes raster, que permitieran no sólo ampliar la información geométrica, sino ser una herramienta que permite superponer capas de información en términos de fachada.

El proceso de desdoblamiento parte de la detección del plano principal de la fachada, sobre el cual se rotará y trasladará las caras de la fachada; el plano principal se entiende como el que mejor describe la fachada dentro de un rango; para su extracción se utiliza el método iterativo RANSAC para la detección del plano más grande que se encuentre en la nube dentro de un rango de $10 \mathrm{~cm}$. Este plano se analiza de forma independiente para obtener una mejor interpretación de las deformaciones que presenta la fachada.

\section{Detección del plano mayor que define la fachada, clasificación semiautomática RANSAC}

La metodología planteada se centra en una nueva forma de reconstruir la información TLS, que parte de la identificación de propiedades físicas de la superficie que compone la fachada, en primer lugar, mediante la extracción del plano que define la mayor superficie de la fachada.

Este proceso se logra mediante el método iterativo RANdomSAmpleConsensus (RANSAC) propuesto por Fischer and Bolles (198I): es un método iterativo para estimar parámetros de un modelo matemático a partir de un conjunto de datos observados.

Proviene del campo de visión por computador y se utiliza principalmente en la fotogrametría para encontrar los puntos correspondientes de un par de imágenes, en el registro de nubes de puntos (Método DARCES) en Chen, Hung y Cheng (1999). También, se utiliza como algoritmo de segmentación geométrica debido a su capacidad para reconocer automáticamente las formas a través de los datos (planos, cilindros, esferas y toros), a pesar del ruido de las mismas.

El paradigma RANSAC extrae al azar un mínimo de puntos base sobre los cuales se busca la relación del punto en este caso al plano. El número mínimo de puntos para el cálculo de 


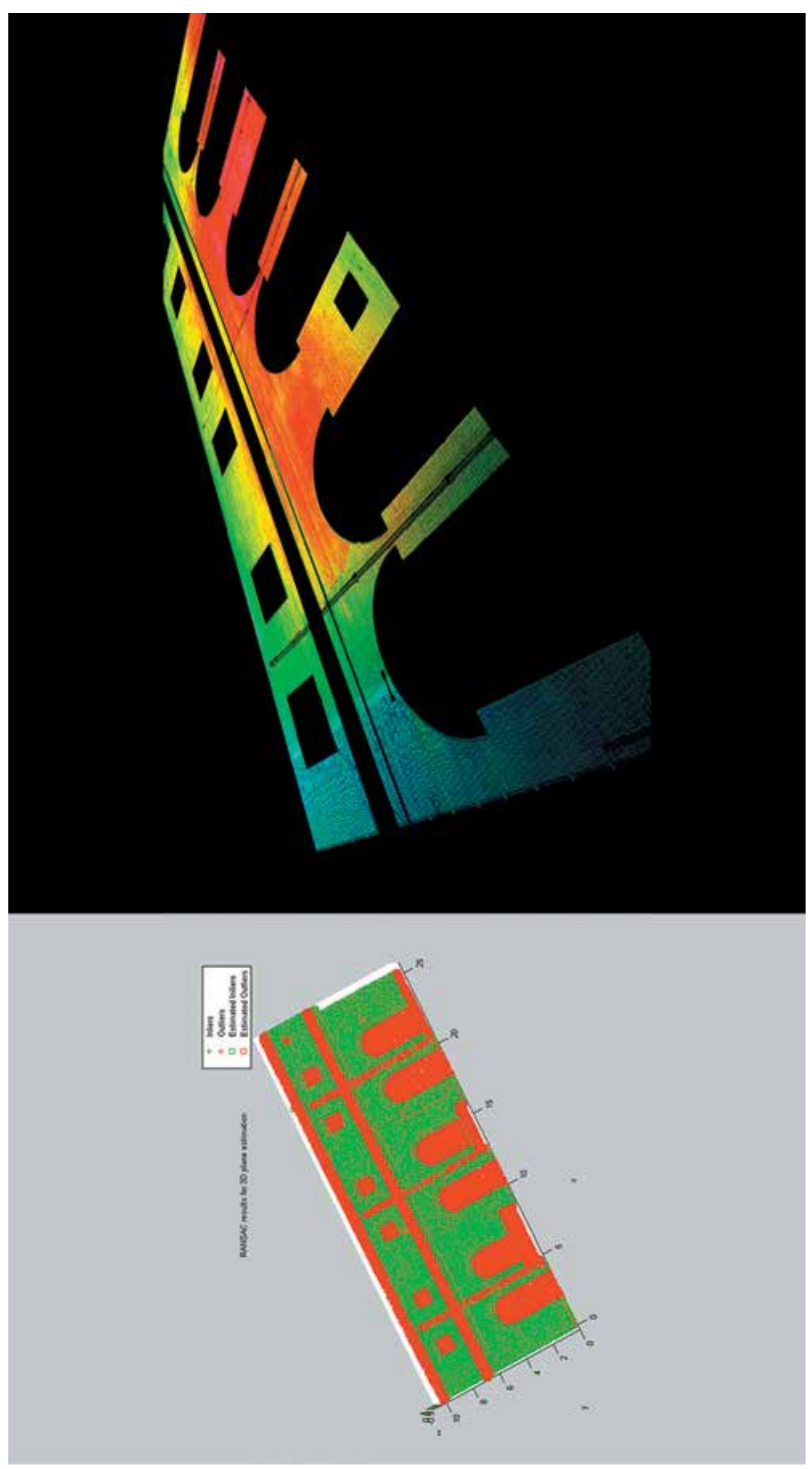

Figura 6. Izquierda extracción de plano de fachada y visualización de puntos extraídos, proceso RANSAC basado en los algoritmos planteados por Kovesi, 2006 y Zuliani, 2012 Fuente: Elaboración propia de los autores. 
la superficie del plano es de 3, siendo un valor muy inferior para casos de estudio TLS, al buscar ajustar un plano a una nube de millones de puntos y sus respectivas combinaciones, convirtiéndose en valores tan altos que imposibilitan una verificación exhaustiva.

Una solución a esta verificación es elegir un subconjunto de puntos no afectados de errores importantes que permitan obtener un ajuste correcto. Para ello, se tiene en cuenta la probabilidad $(P)$ al coger al menos una muestra con todos los puntos no erróneos en relación al número total de observaciones, el número mínimo de puntos para el cálculo $(p)$ de la fracción de observaciones que pueden ser valores atípicos (outliers $\varepsilon$ ) y del número de pruebas seleccionadas de forma aleatoria $(\mathrm{m})$. Dicha probabilidad se puede calcular mediante la siguiente fórmula (Buill, 2009):

$$
m=\frac{\log (1-P)}{\log (1-(1-\varepsilon))^{p}} \quad 8.4 \text { pruebas }=\frac{\log (1-0.99)}{\log (1-(1-0.25))^{3}}
$$

Las formas resultantes son probadas contra los puntos de los datos para determinar cuántos de ellos se aproximan a la forma primitiva (como se observa en la Figura 6). A partir de la extracción de estos datos, se procede a calcular la superficie en busca de encontrar la geometría que define la fachada. Para lograr el promedio, se utilizaron diferentes técnicas en los campos de procesamiento de imágenes y de construcción de mallas, concluyendo que los procesos de mallado permiten un mayor control del promedio de las superficies con procesos de reducción de ruido en mallas que no modifican los límites de la los datos y permiten análisis estadísticos en tiempo real.

\section{Deformación de la fachada y detalle de los materiales}

Partimos de la información clasificada de la fachada en el proceso RANSAC para aislar el plano que define su pandeo o deformación, este plano se promedia quitando todo elemento menor de a los $3 \mathrm{~mm}$ que impida leer la tendencia de las superficies, como se muestra en la metodología de la Figura 7.

La metodología planteada pasa de la nube de puntos a mallas y luego a imágenes, ya que se considera las mallas como un paso intermedio oportuno para no modificar los límites del plano, aun así este proceso se puede llevar a cabo con la conversión directa de puntos a raster, teniendo en cuenta el no modificar o expandir los bordes del plano en relación al tamaño del pixel. En la metodología planteada se filtró la malla, promediando los detalles menores a los tres centímetros, comparando el resultado final con la nube de puntos y evitando sobrepasar una diferencia por desviación estándar de tres centímetros.

Figura 7. Metodología para el análisis geométrico de una fachada considerada plana Fuente: Elaboración propia de los autores

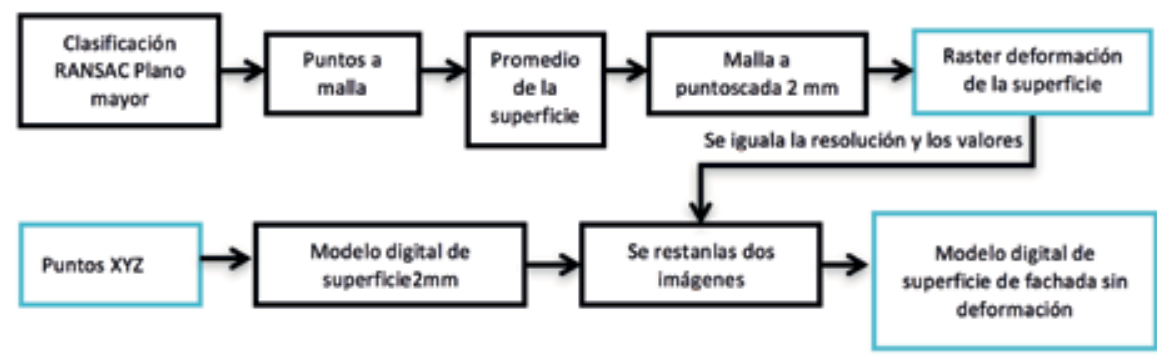

Esta malla se subdivide a cada dos centímetros, generando una nube de puntos que se convertirá en un modelo digital de la superficie de la fachada, este modelo digital de superficie es un raster en $\mathrm{X}, \mathrm{Y}$, que almacena en cada pixel un valor de altura, en este caso la 
altura es en sí el relieve que tiene la fachada. Como el raster es del plano mayor de fachada promediada, se representa como una imagen que describe la deformación de la fachada.

Esta imagen métrica de fachada se resta a la imagen raster generada de forma directa de la nube de puntos TLS, como resultante se obtiene una imagen que destaca los detalles inferiores a tres centímetros en la fachada, en el caso de una fachada de ladrillo visto se ven las juntas de los ladrillos, las fisuras y elementos como grapas. En la Figura 8 se ve la diferencia entre la imagen raster tal cual viene del TLS y la que se le ha restado el plano mayor de fachada promediado, son dos imágenes clasificadas en función al valor de altura en el modelo digital de superficie. En la parte superior de las dos imágenes vemos una sección longitudinal que muestra cómo se elimió la curvatura de la superficie del plano mayor.
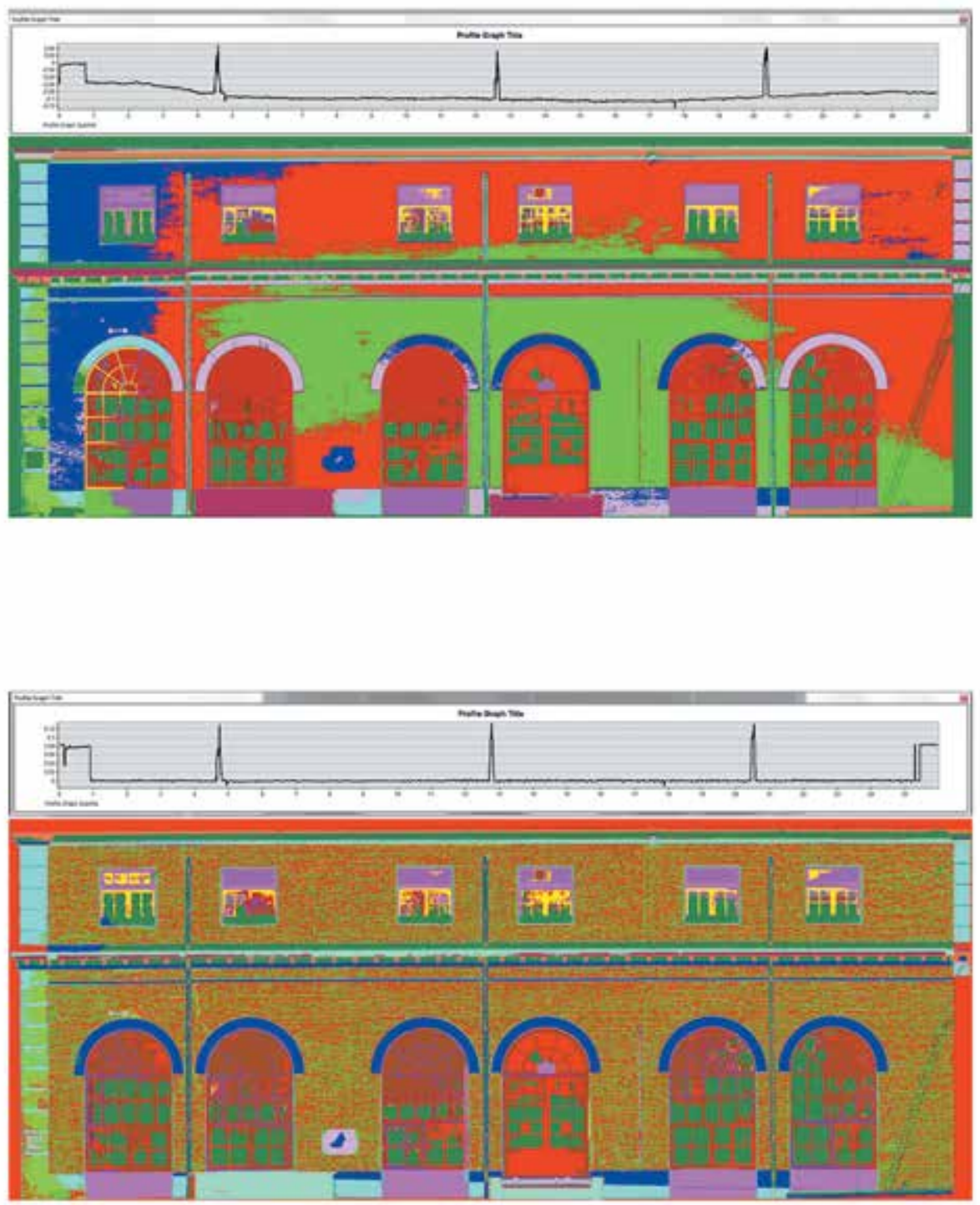

En la Figura 9 se repite esta comparación, destacando cómo la imagen sin pandeo de la superficie permite ampliar el detalle de las fisuras de la fachada, eliminando la deformación que impide ver el valor de profundidad como una información útil para cuantificar una patología concreta.
Figura 8. Clasificación por alturas del modelo digital de superficie proveniente del TLS y la clasificación del modelo digital de superficie sin la deformación de la fachada (Unsupervised 6 ROI)

Fuente: Elaboración propia de los autores 
Figura 9. Modelo digital de superficie generado a partir de la nube de puntos TLS y modelo digital de superficie sin la deformación de la fachada

Fuente: Elaboración propia de los autores.
Figura 10. Análisis de textura SecondMoment del modelo digital de superficie a partir de la información TLS, basada en una matriz Co-ocurrence

Fuente: Elaboración propia de los autores.
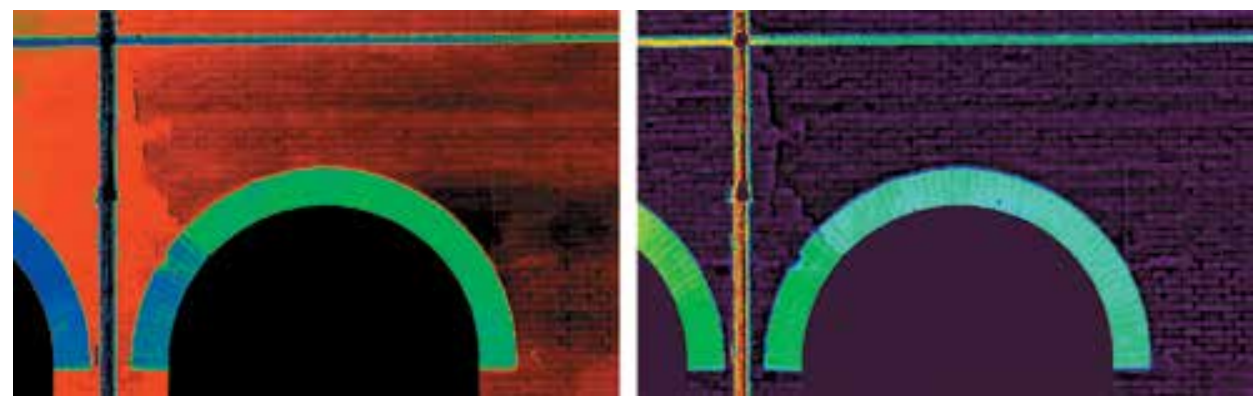

Esta información base requiere una lectura especializada, la comprobación en sitio y estudios complementarios, dada la complejidad de estudios que se pueden realizar de una fachada patrimonial que involucran grados de conocimiento e interpretación de diferentes disciplinas.

\section{Límites de los elementos que definen la fachada}

Desde el punto de vista geométrico, la identificación de las aristas tiene un interés creciente como información de soporte para la definición de elementos arquitectónicos. Entendiendo que la expresión gráfica en la arquitectura "no debe entenderse como una simple actividad técnica o una destreza mecánica; sino como una tarea de análisis y síntesis, que requiere iniciativa personal y está llena de intenciones” (Gámiz, 2003).

En este proceso, la tecnología juega un papel cada vez más importante y aunque tenga una variante técnica relevante, requiere a su vez una síntesis con objetivos claros, siendo necesaria la incorporación de términos de estandarización que permitan un mayor grado de entendimiento entre disciplinas.

Partiendo de esta premisa, se busca optimizar la información TLS para llegar a describir la fachada con líneas que defina sus aristas y modificando el valor de línea de estas en función a la capa que las identifica, sin llegar a pretender imitar un plano arquitectónico, sino como una base para llegar a un mayor grado de detalle planímetro en fachadas.

Partimos del análisis de textura basada en una matriz Co-occurrence, específicamente el proceso Second moment, identificando límites de los elementos en relación a la profundidad, teniendo como base el tamaño del pixel de medio centímetro y la relación de pixel vecinos $3 \times 3$ pixeles.

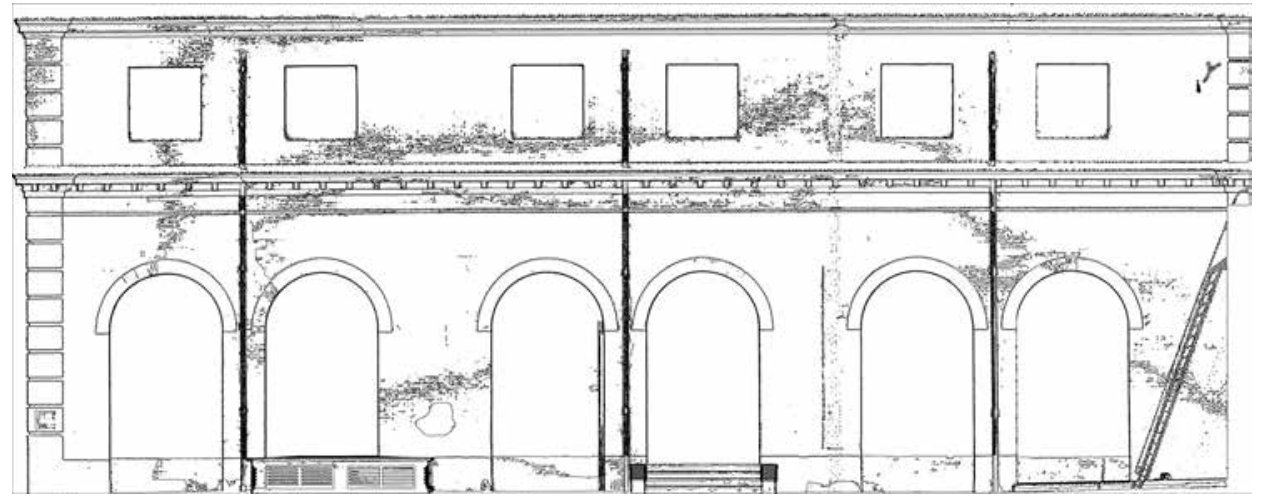

Este proceso se aplicó tanto en el modelo digital de superficie de la información TLS sin filtros, como en el modelo digital de superficies sin pandeo descrito en el apartado 4.3.2. 
En el primer proceso, la Figura 10 muestra la afectación del pandeo de la superficie, la cual coincide con diferentes puntos de fisura de la superficie, teniendo en cuenta que la parte superior de la fachada en la que se denota más este pandeo no es una ampliación de la fachada, como se explicó anteriormente, debido a la necesidad de aumentar las caladeras de carbón a gasoil. La Figura I I que corresponde al análisis de textura Second Moment del modelo digital de superficie sin deformación, no tiene la línea de quiebre de la deformación de la fachada, permitiendo una lectura clara de los elementos que la componen.

En ambos casos, los cambios más drásticos de la superficie en relación a la profundidad tienen un valor de línea mayor, existiendo un salto de información entre la información y el "no data", generando errores fácilmente identificables visualmente, por ejemplo en la zona derecha, producida por la oclusión de una escalera. Este error se puede reducir con una máscara que contenga el "no data", en la Figura I I este error se minimiza por el promedio de la superficie del plano mayor.

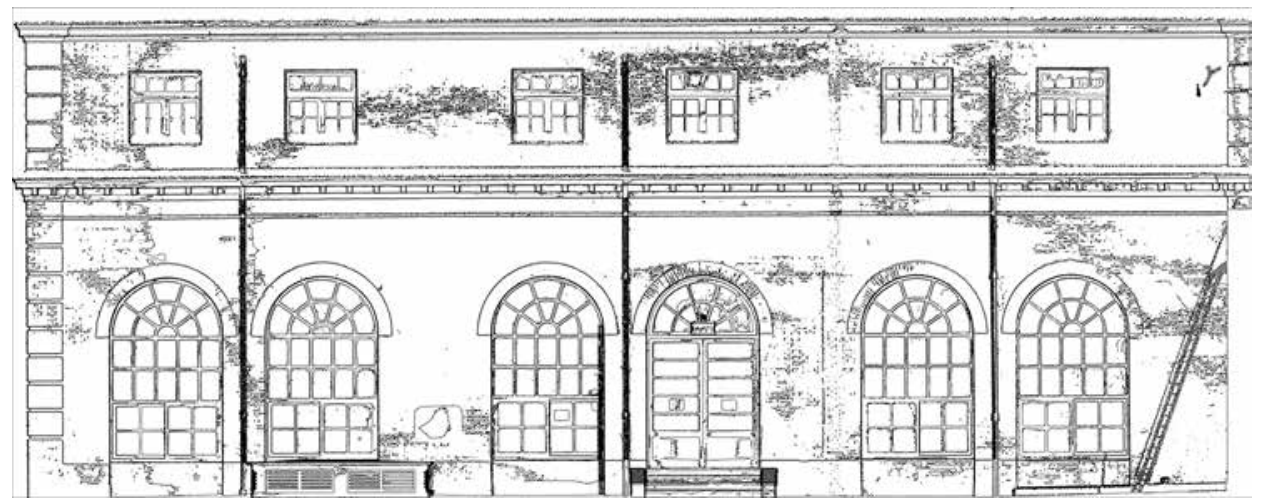

\section{LÍMITES DE LOS MATERIALES DE FACHADA}

La identificación de los materiales de fachadas es una tarea compleja. "Hay materiales de gran importancia arquitectónica que arqueológicamente apenas tienen relevancia. Hay materiales de una importancia histórica fundamental que arquitectónicamente pueden ser deleznables" (González-Moro, 20II). Se utiliza el término "unidad estratigráfica arquitectónica" para estudiar zonas de las fachadas a partir del material, se puede definir la unidad estratigráfica como una zona homogénea que permite una lectura, tanto de construcción, demolición o transformación del bien patrimonial. Las unidades estratigráficas se distinguen entre ellas por sus materiales y técnicas constructivas, por su color, composición, acabado, labra superficial, entre otras ( Mlleto y Vega, 20II).

A continuación se propone una metodología para destacar parámetros geométricos de la textura del material en la fachada, entendiendo que para su adecuada interpretación hay que hacer un estudio complementario in situ.

\section{Análisis de curvatura del material de fachada}

Los análisis aplicados sobre el modelo digital de superficie provienen de técnicas usadas en Modelos Digitales de Terreno DTM, entre estos análisis considerados topográficos se encuentra el proceso Shader Relief (ver Figura 12), que mediante la simulación de un azimut y ángulo de una fuente de luz, permite recrear una iluminación sobre el modelo digital. Con lo anterior, se consigue visualizar el acabado de la superficie, teniendo en cuenta la relación de los pixeles vecinos, que en este caso es de 2 pixeles, buscando la visualización de las aristas más pequeñas, teniendo en cuenta que el valor de cada pixel del raster es de medio centímetro.
Figura I I. Análisis de textura Second Moment de modelo digital de superficie sin deformación, basada en una matriz Coocurrence

Fuente: Elaboración propia de los autores. 
Figura 12. Filtro Shader Relief. Izquierda: nube de puntos e imagen Shader Relief en detalle. Derecha: imagen Shader Relief

Fuente: Elaboración propia de los autores.
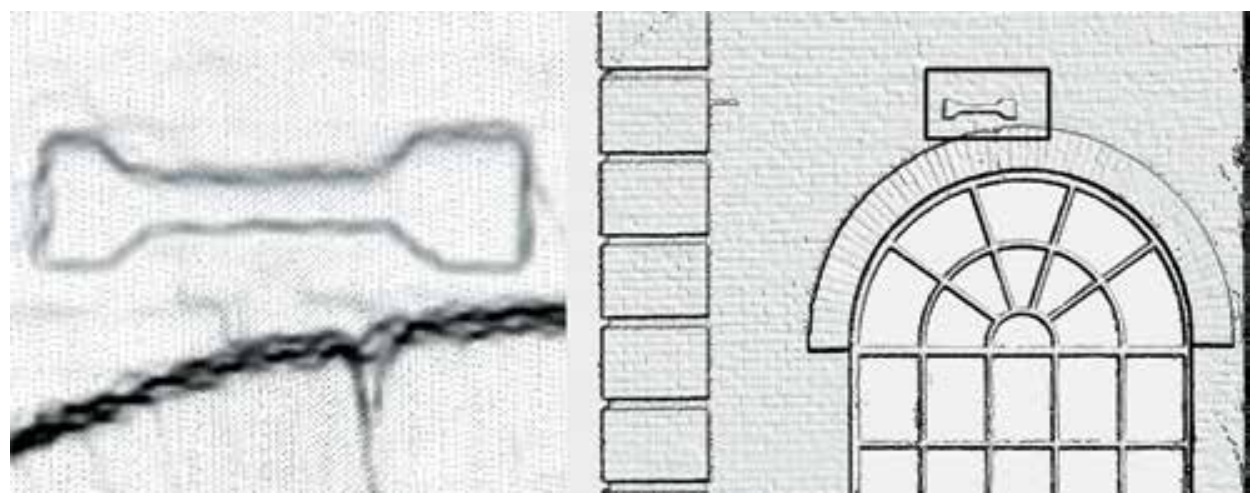

Esta información se considera necesaria para delimitar elementos de pequeña escala. A la izquierda de la Figura 12 hay dos tipos de información superpuesta (visualizadas desde autocad y con una extensión de pointools para nubes de puntos): la imagen Shades Relif y la nube de puntos, en ésta se puede ver que la información de puntos es ilegible haciendo zoom sobre la zona de interés, mientras que la imagen raster sí permite esta aproximación. La misma situación se repite cuando se genera la imagen directamente en visualizadores de nubes de puntos, ya que para su correcta visualización tienen que superponerse la información de los puntos, la cual no es homogénea, afectando la escala y el detalle al que puede llegar la información TLS.

La imagen generada a partir de análisis de la topografía de la superficie, permite promediar el límite entre elementos complejos como por ejemplo bordes en pendiente, simplificando dicha información en imágenes que permiten ver cambios en la superficie. Cabe destacar que esta información requiere una base que minimice el ruido de puntos fuera del promedio de las superficies, ya que este ruido se ve aumentado en análisis, se recomienda la utilización de filtros en las imágenes de profundidad, como los filtros de media que eliminen pixeles aislados fuera del promedio de la superficie.

\section{CONCLUSIONES}

La información TLS permite el análisis geométrico de fachadas y de los materiales que la componen, este análisis se puede llevar hacer tanto en 3D como en 2,5D y 2D, teniendo como ventaja que los análisis en dos dimensiones se pueden sobreponer, complementando la información RGB y la intensidad con la información geométrica y análisis de la fachada.

La clasificación de la nube de puntos en 3D permite una mayor difusión y comprensión del bien. Igualmente, la clasificación de la información en dos dimensiones permite descomponer en capas elementos específicos del edificio, sin descartar la relación con otras informaciones, permitiendo contrastar y ampliar la información.

De la misma manera, el filtrado de zonas clasificadas permite nuevas aproximaciones al bien, como se demostró al promediar el plano mayor, destacando características del material y del pandeo de la superficie, reduciendo los procesos manuales - visuales (naked eye), generando bases de datos estándares, teniendo como valor añadido el poder comparar estos análisis en distintos levantamientos del mismo edificio, en distintas fechas y estados de conservación.

\section{Trabajo futuro}

Profundizar en las unidades estratigráficas de la arquitectura patrimonial, de forma objetiva, argumentada y subjetiva, con una lectura crítica de valores históricos y artísticos de los elementos estudiados. 
Desde el punto de vista técnico, comparar características técnicas del mismo procedimiento con otros casos de estudio teniendo en cuenta: densidad de datos/distancia, ángulo de incidencia, características del equipo, condiciones de iluminación, comparar TLS (ajustes y rendimiento necesario), precisión/exactitud, rendimiento alcanzado. A partir de ello, se identifica la fiabilidad de los resultados y los limitantes del método propuesto en relación a los casos propuestos, concretando las tipologías de análisis.

\section{REFERENCIAS}

Aparicio, M. (20II). El Modelo Barcelona de Espacio Público y Diseño Urbano: Producción cultural como motor de desarrollo urbano. El caso del programa "Fàbriques de Creació" de Barcelona. [Tesis de máster]. Universitat de Barcelona, Facultad de Bellas Artes, Máster en Diseño Urbano: Arte, Ciudad, Sociedad, Barcelona, pp. II4-I25.

Buill, F. (2009). Caracterización geométrica con técnicas ópticas 3D aplicadas al modernismo en Cataluña. [Tesis doctoral]. Comunicación visual en arquitectura y diseño. BarcelonaTech: Universitat Politècnica de Catalunya.

Chen, C., Hung, y, Cheng, J. (1999). RANSAC-based DARCES: A New Approch to fast Automatic Registration of Partially Overlapping Gande Image. In: IEEE Transacrions on Ptattern Analysis and Machine Intelligence, 2 I (I I), 1229-I 234.

Gámiz, G. (2003). Ideas sobre análisis, dibujo y arquitectura. Sevilla: Universidad de Sevilla, Secretariado de Publicaciones.

González-Moro, P. (20II). La conservación del tiempo en la restauración: el proyecto estratigráfico, en Arqueología aplicada al estudio e interpretación de edificios históricos, Últimas tendencias metodológicas (pp.42-49.). España: Ministerio de Cultura,

Kovesi, P. (2006). MATLAB and Octave Functions for Computer Vision and Image Processing.

Letellier, R. (2007). Recording, Documentation, and Information Management for the Conservation of Heritage Places, Guiding principles. Los Angeles: The getty Conservation Institute, pp. $45-56$

Lerma, J. (2008). Implementation of an architectonic gis on a brickwork farmhouse, ISPRS Congress, The International Archives of the Photogrammetry. In: Remote Sensing and Spatial Information Sciences, 37, 1013-1015, Part B5, Beijing.

Mileto, C., Vegas, F. (20I I). El análisis estratigráfico: una herramienta de conocimiento y conservación de la arquitectura, en Arqueología aplicada al estudio e interpretación de edificios históricos, Últimas tendencias metodológicas. España: Ministerio de cultura.

Simon, C. (2010). Integration of high-resolution spatial and spectral data acquisition systems to provide complementary datasets for cultural heritage applications. San Jose, United States: published in "IS\&T/SPIE Electronic Imaging meeting,

Zuliani, M. (2012). RANSAC for Dummies With examples using the RANSAC toolbox for Mat$l a b^{\mathrm{TM}}$ \& Octave and more. 